\title{
Reactive oxygen species and age-related genes p66Shc, sirtuin, FoxO3 and klotho in senescence
}

Igor Afanas'ev

Vitamin Research Institute; Moscow, Russia
Key words: ROS signaling, genes, senescence, aging

Submitted: 11/26/09

Revised: 12/26/09

Accepted: 12/28/09

Previously published online: www.landesbioscience.com/journals/ oximed/article/11050

Correspondence to: Igor Afanas'ev;

Email: iafananizer@gmail.com
$\mathbf{R}$ eactive oxygen species (ROS) superoxide and hydrogen peroxide perform important signaling functions in many physiological and pathophysiological processes. Cell senescence and organismal age are not exemptions. Aging-regulating genes $p 66 s h c$, Sirtuin, FOXO3a and Klotho are new important factors which are stimulated by ROS signaling. It has been shown that ROS participate in initiation and prolongation of gene-dependent aging development. ROS also participate in the activation of protein kinases Akt/PKB and extracellular signal-regulated kinase ERK, which by themselves or through gene activation stimulates or retards cell senescence. Different retarding/stimulating effects of ROS might depend on the nature of signaling species-superoxide or hydrogen peroxide. Importance of radical anion superoxide as a signaling molecule with "super-nucleophilic" properties points to the possibility of the use of superoxide scavengers (SOD mimetics, ubiquinones and flavonoids) for retarding the development of aging.

Cellular (replicative) senescence and organismal senescence (the aging of a whole organism) are characterized by the declining ability of cells to respond to oxidative stress and correspondingly increase the risk of aging-associated diseases. It has been supposed that senescent cells accumulate with age and by this contribute to organismal aging. Such a view on the development of human aging was accepted by many authors and instigated the search for methods to suppress cellular senescence. However, it should be noted that the programmed mechanisms of senescence and apoptosis might prevent the conversion of normal cells into potential cancer cells by committing suicide (apoptosis) or irreversibly arresting growth (senescence) rather than replicate.

Numerous studies demonstrate an important role for reactive oxygen species (ROS) in the development of senescence and aging (Harman's free radical theory of aging). There are different sources of superoxide, a precursor of other ROS in cells such as mitochondria, xanthine oxidase, NADPH oxidase and NO synthases. ${ }^{1,2}$ It is usual practice to apply antioxidants to reduce ROS damaging effects, however current studies demonstrate that ROS possess another apparently more important function: to be signaling molecules in various enzymatic and gene-mediated physiological and pathophysiological processes. In addition, the suppression of cellular senescence can enhance the possibility of cellular transformation in immortal cancer cells. Therefore, ROS-initiated apoptosis (programmed cell death) might result in an increase in longevity for the whole organism. Therefore, ROS signaling might be important in both suppression and stimulation of aging development.

\section{Different Signaling Functions of Superoxide and Hydrogen Peroxide}

Conflicting effects of ROS can depend on many factors including ROS chemical structures. ROS superoxide $\mathrm{O}_{2}{ }^{--}$and hydrogen peroxide $\mathrm{H}_{2} \mathrm{O}_{2}$ are major regulators and signaling molecules in cellular senescence. They belong to different 


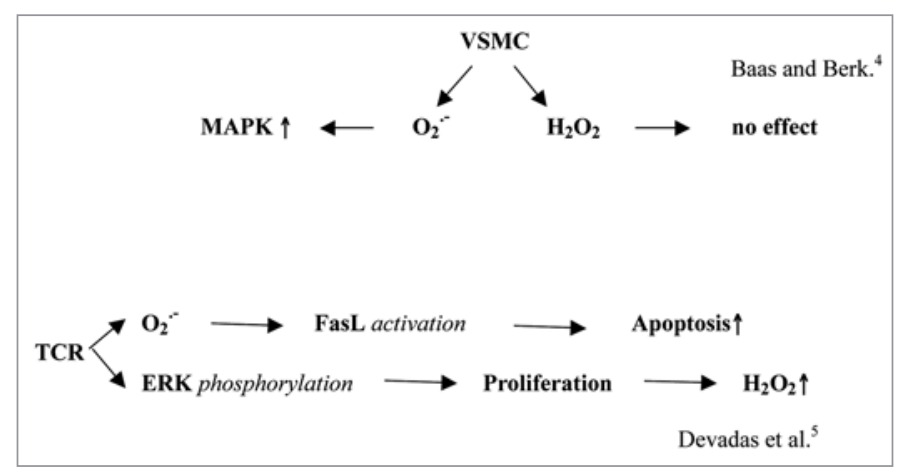

Figure 1. Different signaling effects of superoxide and hydrogen peroxide on enzymatic processes.

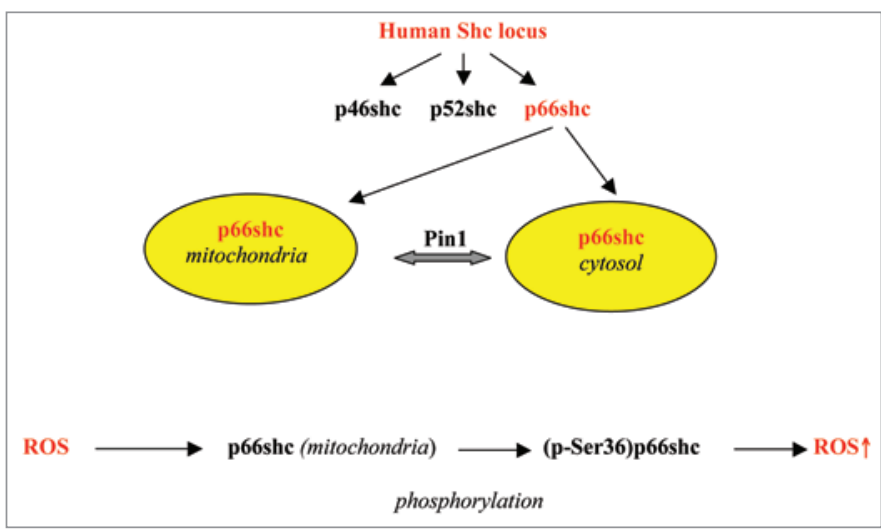

Figure 2. Pinton's mechanism of ROS signaling in the activation of p66shc protein. ${ }^{10}$

classes of chemical compounds: superoxide is a paramagnetic free radical anion and hydrogen peroxide is a diamagnetic molecule. Therefore, their signaling and regulatory functions might be different. Superoxide is a "super-nucleophile," and is able to participate in phosphorylation/ dephosphorylation of protein kinases. ${ }^{3}$ Hydrogen peroxide can directly inhibit protein phosphatases and by this activate protein kinases, but possibly more importantly hydrogen peroxide is able to stimulate superoxide-producing enzymatic reactions.

Many experimental findings confirm the different effects of superoxide and hydrogen peroxide on numerous enzymatic processes including apoptosis and proliferation. For example, it has been shown that superoxide increased mitogenactivated protein kinase (MAPK) activity in cultured rat aortic vascular smooth muscle cell (VSMC) while hydrogen peroxide had no effect. ${ }^{4} \mathrm{~T}$ cell receptor (TCR) signaling activates two distinct pathways of ROS generation: hydrogen peroxide regulated extracellular signal-regulated kinase (ERK) phosphorylation (proliferative pathway) and superoxide mediated TCR-stimulated activation of the pro-apoptotic Fas ligand (FasL) promoter and subsequent cell death ${ }^{5}$ (Fig. 1). There are also other numerous examples of different effects of superoxide and hydrogen peroxide signaling in cellular processes. ${ }^{6-8}$ It is therefore possible that, in part at least, the contradictory effects of ROS on aging processes depend on whether superoxide or hydrogen peroxide are the signaling molecules in these processes.

\section{Signaling by Reactive Oxygen Species in Gene- and Enzyme-Mediated Age Development}

p66shc gene. It was demonstrated in 1999 that targeted mutation of the mouse p66shc gene induced stress resistance and prolonged lifespan in experimental animals. ${ }^{9}$ For example, p66shc $\mathrm{c}^{-/}$mice increased resistance to oxidative stress and resulted in a 30\% increase in lifespan. Further studies revealed the mechanism of p66shc activity.

It was agreed that the favorable effect of suppression of $p 66 \mathrm{shc}$ gene was due to its pro-oxidant activity. Shc locus encodes three adaptor proteins (p46shc, p52shc and p66shc) but only p66shc is regulated by ROS signaling. p66shc presents in both mitochondria and cytosol but only in mitochondria this enzyme is activated by ROS through the phosphorylation of a critical serine residue Ser-36 (Fig. 2). Pinton et al. ${ }^{10}$ has shown that phosphorylation is catalyzed by protein kinase $C \beta$ which in turn was activated by ROS. Activation of mitochondrial p66shc shifts equilibrium between mitochondria and cytosol to the left catalyzed by prolyl isomerase Pin1 (Fig. 2). This gene/enzymatic cascade results in a further increase in ROS formation. Accumulation of p66shc in mitochondria changes mitochondrial calcium responses and induces apoptosis. These data show a new signaling route that activates an apoptotic inducer and shortens the life span.

Gertz et al. ${ }^{11}$ proposed another mechanism of ROS signaling that resulted in the activation of p66shc protein (Fig. 3). These authors believe that under normal physiological conditions p66shc presents in reduced form in the cytosol. ROS activate p66shc by oxidation of sulfhydryl groups to disulfide bridge and import it to mitochondrial intermembrane space (IMS). If IMS contains sufficient levels of reductants [supposedly glutathione (GSH) and thioredoxcins (Trx)], they reduce p66shc into the inactive reduced form. If contents of reductants are small, then the activated oxidized p66shc protein generates ROS and initiates apoptosis.

It has also been suggested that ROS can activate $\mathrm{p} 66 \mathrm{shc}$ by the dissociation of p66shc-heat shock Hsp70 protein inhibitory complex in mitochondria and initiate apoptosis. ${ }^{12}$ Trinei et al. ${ }^{13}$ show that p53-p66shc signaling pathway regulates ROS formation, levels of oxidation-damaged DNA and ROS-induced apoptosis. Giorgio et al. ${ }^{14}$ pointed out that p66shc 
is a redox enzyme that generated mitochondrial hydrogen peroxide acting as a signaling molecule for apoptosis (Fig. 4A). These authors also suggested that p66shc is able to withdraw electrons from the mitochondrial electron transfer chain by the oxidation of cytochrome $c$. Nemoto et al. ${ }^{15}$ proposed that $\mathrm{p} 66 \mathrm{shc}$ may regulate mitochondrial metabolism by the partition of ATP generation in the cells. In the absence of $\mathrm{p} 66 \mathrm{shc}$, the mitochondrial oxidative pathway is reduced, whereas glycolysis is increased.

A critical step of p66shc activation is the phosphorylation of its Ser-36 residue. This process depends on the production of ROS by various sources such as the advanced glycation end products (AGEs), hyperglycemia (HG), or mitochondrial protein kinase $C \beta$ (Fig. 4B). It has been shown that AGEs stimulate ROS formation in diabetes and agingrelated diseases. Cai et al. ${ }^{16}$ investigated the effects of AGEs in human embryonic kidney cells (HEK293). Stimulation of HEK293 cells with AGEs caused Ser-36 phosphorylation of p66shc and increased the phosphorylation of protein kinase Akt/B and FOXO3a by approximately threefold. AGE-induced phosphorylation of FOXO3a led to the inhibition of MnSOD which was strongly inhibited by an antioxidant (N-acetylcysteine). This study demonstrates a new pathway for prooxidant action of AGEs through Ser36 phosphorylation of p66shc, FOXO3a inactivation, and MnSOD suppression in human kidney cells.

Malhotra et al. ${ }^{17}$ found that the high glucose (HG)-stimulated adult rat ventricular myocytes exhibited a marked increase in ROS production, upregulation of (phospho-Ser-36)-p66shc, collapse of mitochondrial transmembrane potential, and increased formation of $\mathrm{p} 66 \mathrm{shcA} / \mathrm{cyto}$ chrome $c$ complexes. These indices of oxidative stress were accompanied by a $40 \%$ increase in apoptosis and the upregulation of cleaved caspase- 3 and the apoptosisrelated proteins $\mathrm{p} 53$ and $\mathrm{Bax}$.

It was found that the Ser-36-phosphorylated form of p66shc increased with the age of experimental animals. ${ }^{18}$ The level of Ser-36 phosphorylation of p66shc continually enhanced in old mice. Increase in p66shc phosphorylation at

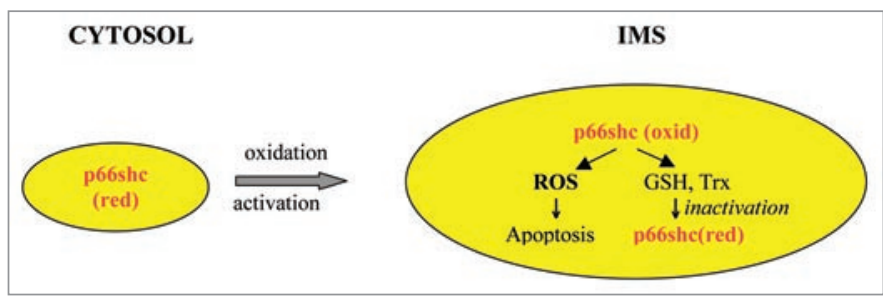

Figure 3. Gertz's mechanism of ROS activation of p66shc protein."

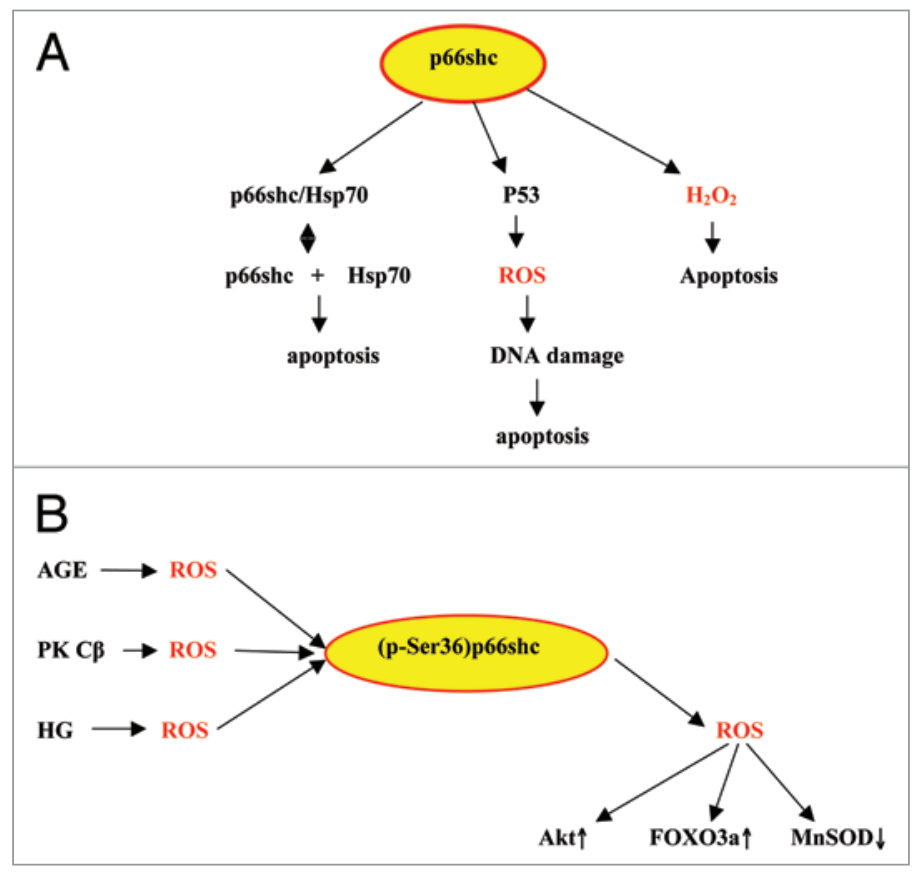

Figure 4. (A) Regulation by p66shc of ROS-induced apoptosis (Orsini et al. ${ }^{12}$ Trinei et al. ${ }^{13}$ and Giorgio et al. ${ }^{14}$ ). (B) ROS-mediated phosphorylation of p66shc and ROS signaling pathways of activation of FOXO3a and Akt kinase and suppression of MnSOD (Pinton et al. ${ }^{10}$ Cai et al. ${ }^{16}$ and Malhotra et al. ${ }^{17}$ ).

Ser-36 caused higher free radical production and consequently the accumulation of damages by free radicals in age development. The increased amount of Ser-36phosphorylated p66shc in livers of 12- and 23-month-old mice was correlated with the decreased level of antioxidant enzymes. Khanday, et al. ${ }^{19}$ suggested that Ser-54 and Thr-386 are novel phosphorylatable residues in p66shc that regulated rac-induced increase and mediated racl-stimulated cellular oxidative stress and death.

Activation of p66shc and ROS generation are the starting point of various pathologies. Husain et al. ${ }^{20}$ investigated a role for p66shc protein in HIV-1-induced ROS generation and apoptosis of immortalized differentiated human podocytes
(CIDHP). CIDHP transfected with truncated HIV-1 construct (NL4-3) exhibited the increased ROS formation, DNA strand breaks and a five-fold increase in apoptosis. It was concluded that inhibition of phosphorylation at Ser-36-p66shc prevented the generation of HIV-1 stress signals and activation of the CIDHP apoptosis program.

It is known that ROS and insulin signaling in the adipose tissue stimulate aging and age-associated diseases. Berniakovich et al. ${ }^{21}$ found that insulin activated p66shc in adipocytes and that p66shc-generated ROS regulated insulin signaling through Akt (protein kinase B) phosphorylation and FOXO3a. Deletion of p66shc resulted in increased mitochondrial uncoupling 


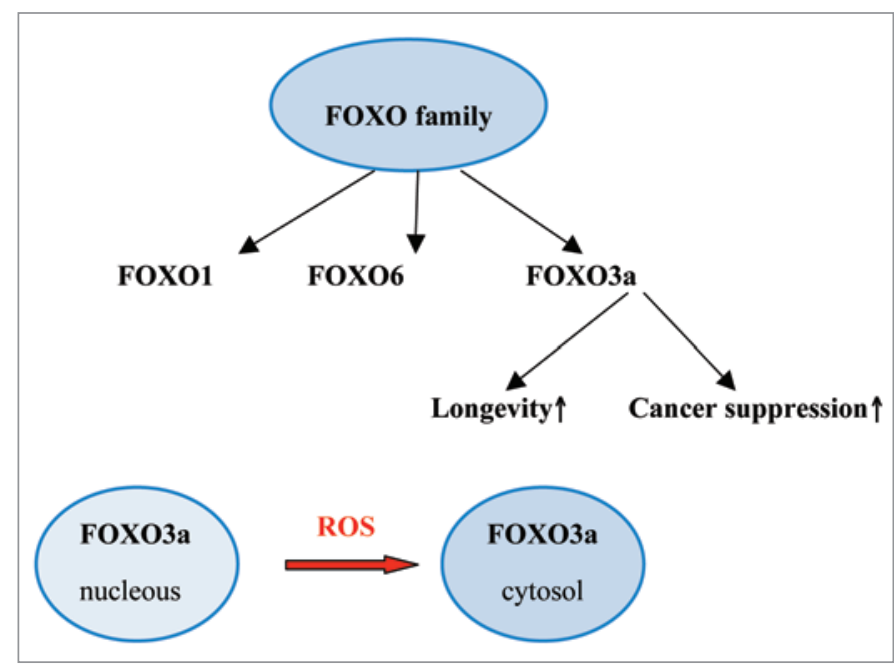

Figure 5. Forkhead family of transcription factors.

and reduced triglyceride accumulation in adipocytes and in vivo increased metabolic rate and decreased fat mass and resistance to diet-induced obesity.

It has been found that NADPH oxidase in macrophages of p66shc-knockout mice p66shc(-/-) mice decrease superoxide production by $40 \% .{ }^{22}$ The rate of phosphorylation of $\mathrm{p} 47$ phox decreased in mutants, as well as the membrane translocation of this complex. It was possibly due to a decrease in the phosphorylation of protein kinasee $\mathrm{C} \delta$, Akt/B and ERK that was responsible for the phosphorylation of $\mathrm{p} 47 \mathrm{phox}$.

It has been noted above that Shc locus encodes three adaptor proteins (p46shc, p52shc and p66shc) but only p66she is regulated by ROS signaling. However, Arany et al. ${ }^{23}$ demonstrated that all three proteins can participate in the regulation of oxidative stress. They showed that tyrosine-phosphorylated p46shc and $\mathrm{p} 52$ shc proteins activated epidermal growth factor receptor EGFR and correspondingly the EGFR/Ras/MEK/ERK survival pathway, whereas the p66shc protein inhibited their functions. Thus in order to ameliorate oxidative stressinduced cell injury it might be useful to inhibit Ser-36 phosphorylation of p66shc or knock down p66shc expression in vivo.

$F O X O 3 a$ gene. FOXO3a belong to the O subclass of the forkhead family of transcription factors which are characterized by a fork head DNA binding domain. There are three main proteins (FOXO1, FOXO3a and $\mathrm{FOXO} 4$ ) from which $\mathrm{FOXO} 3$ a protein is considered to be a regulator of longevity and cancer (Fig. 5). However, the other FOXO proteins, for example FOXO1, are apparently also capable of participating in ROS-dependent cascades (see below). It has also been proposed that $\mathrm{FOXO} a$ phenotype is associated with a longer life in humans. ${ }^{24}$ In mammal cells, FOXO3a is predominantly in the nucleus, but ROS initiates its redistribution to the cytosol. ${ }^{25}$ Miyauchi et al. ${ }^{26}$ proposed that FOXO3a plays an important role in the enzymaticgene cascade insulin/insulin-like growth factor-1 (IGF-1)/phosphatidylinositol-3 kinase (PI3K)/Akt/FOXO3a. In mammalian cells the activation of this signaling pathway leads to senescence. Guo et al. ${ }^{27}$ showed that p66shc participates in an $\alpha_{1}$-adrenergtic receptor $\left(\alpha_{1}\right.$-AR) pathway that requires the cooperative effects of protein kinases PKC $\varepsilon$ and PKC $\delta$ and leads to Akt-FOXO3a phosphorylation in cardiomyocytes. $\alpha_{1}$-AR stimulated ROSdependent p66shc phosphorylation that required epidermal growth factor receptor (EGFR) and PKCe activity. p66Shc linked $\alpha_{1}$-AR to an Akt signaling pathway that selectively inactivated FOXO3a and downregulated mitochondrial MnSOD. Thus this and other studies demonstrate that ROS phosphorylation/activation of $\mathrm{FOXO} 3 \mathrm{a}$ resulted in cellular senescence. ${ }^{27-29}$

It is known that AGEs are elevated in aged and diabetic individuals. Alikhani et al. ${ }^{30}$ demonstrated that proapoptotic transcription factor FOXO1 stimulated $\varepsilon$-(carboxymethyl)lysine (CML)-collageninduced fibroblast apoptosis. Inhibition of p38 and JNK kinases reduced CMLcollagen-stimulated apoptosis while the inhibition of the phosphatidylinositol 3-kinase/Akt pathway enhanced FOXO1 activation. ROS suppression blocked p38 and JNK stimulation by CML-collagen. These findings showed that FOXO1 was activated by the ROS-depended activation of p38 and JNK and inactivated by Akt kinase.

It was also showed that the insulininduced PI3K/Akt activation pathway inhibited a family of forkhead transcription factors (FOXO) resulting in an increase in ROS formation in cells. Kim et al. ${ }^{31}$ studied the interaction of FOXO1 with proinflammatory cytokine NFKB. It was found that the treatment of cells with insulin led to NFKB activation through the phosphorylation of FOXO1 by PI3K/ Akt pathway. It was suggested that during aging the phosphorylation of FOXO1 regulated NFKB nuclear translocation by activating PI3K/Akt.

Li et al. ${ }^{32}$ studied MnSOD expression and its transcriptional regulation in vascular smooth muscle cells (VSMC) isolated from old versus young rats grown in normal or high glucose or at different TNFo levels. MnSOD activity was reduced in VSMC from old compared with young animals. Inhibition of $\mathrm{FOXO} 3$ a transcription led to a reduction in $\mathrm{MnSOD}$ gene expression. VSMC from old rats increased the phosphorylation of FOXO3a at Ser253 which paralleled the reduction of MnSOD protein. Treatment of cells with IGF-1 induced phosphorylation of Akt and FOXO3a. Akt activity increased in VSMC from old rats and by this initiated the phosphorylation and inactivation of FOXO3a and downregulation of MnSOD transcription.

In subsequent work, Li et al..$^{33}$ studied the effect of insulin-like growth factor-1 receptor (IGF-1R) on the Akt/FOXO3a and ERK pathways in VSMC from young and old rats. They found the constitutive activation of IGF-1R in VSMC from old compared to young rats. IGF-1R signaling modulated MnSOD and catalase genes through activation of the Akt/FOXO3a pathway. Activation of IGF-1R signaling influenced VSMC function in old rats and 
may contribute to the increased risk for atherosclerosis. It has been proposed that a decrease in FOXO3a expression is responsible for age-accelerated atherosclerosis in old mice. ${ }^{34}$

Tan et al. ${ }^{35}$ proposed that FOXO3a is able to inhibit cardiomyocyte hypertrophy through the transactivation of catalase. Nakamura and Sakamoto showed that hydrogen peroxide stimulated the expression and phosphorylation of FOXO resulting in the induction of luteal cell apoptosis. ${ }^{36}$ It was found that hydrogen peroxide induced $\mathrm{FOXO} 3 \mathrm{a}$ accumulation in the nucleus causing the transactivation of IRS promoter activity and ROSinduced apoptosis in mammalian cells. As it follows from already considered data, p66shc and FOXO3a may participate jointly in ROS stimulation. Chintapalli et al. ${ }^{37}$ showed that the inhibition of p66shcA in mesangial cells prevented glycooxidantdependent FOXO3a regulation and promoted the survival phenotype.

It was found that that the increased flux of free fatty acids (FFA) in adipocytes increased mitochondrial ROS production and decreased the levels of FOXO1 and transcription of antioxidant enzymes MnSOD and glutathione peroxidase. ${ }^{38}$ Resveratrol protected cells from FFAinduced ROS formation by increasing FOXO1/Sirt1-dependent MnSOD and glutathione peroxidase activation. (Some FOXO-depended enzymatic pathways are presented in Fig. 6).

Gene family SIRTUIN. Silent information regulator Sirtuin (human Sirt1 and Sirt3 and yeast Sir2) proteins are deacetylases hydrolyzed acetyl-lysine residues. Sirt 2 protein mediates longevity of yeast but the effect of Sirl on the lifespan of mammals is still uncertain. Alcendor et al. ${ }^{39}$ showed that Sirt1 was significantly upregulated in response to low/moderate oxidative stress in nontransgenic adult mouse hearts. This Sirt1 upregulation suppressed age-dependent cardiac hypertrophy, apoptosis, cardiac dysfunction, and expression of senescence markers. In contrast, a high level of Sirt1 enhanced these damaging disorders. Moderate overexpression of Sirt1 protected from ROS overproduction by paraquat and increased the expression of catalase through FOXO3a-dependent mechanisms, while

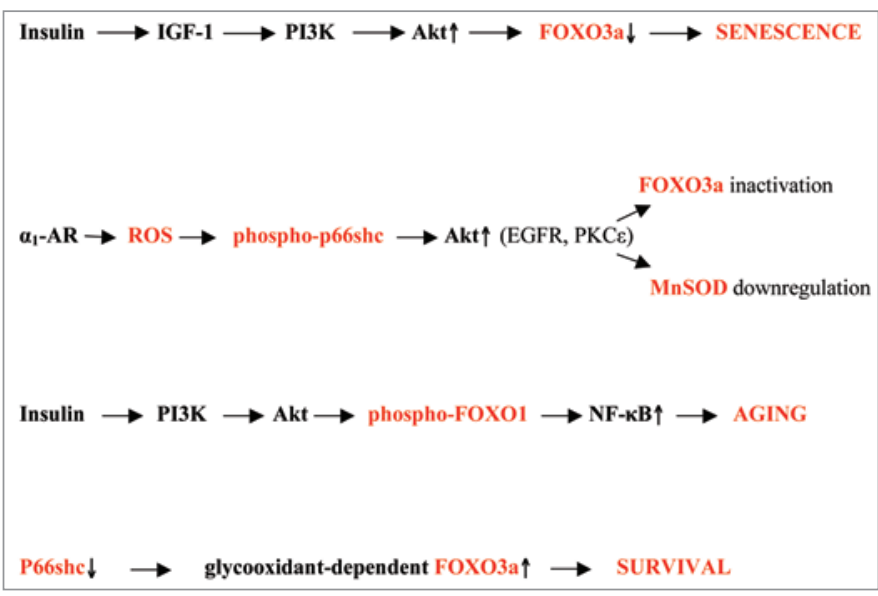

Figure 6. ROS-depended gene/enzyme cascades leading to activation/inactivation FOXO genes (Miyauchi et al., ${ }^{26}$ Guo et al. ${ }^{27}$ and Purdom and $\mathrm{Chen}^{28}$ ).

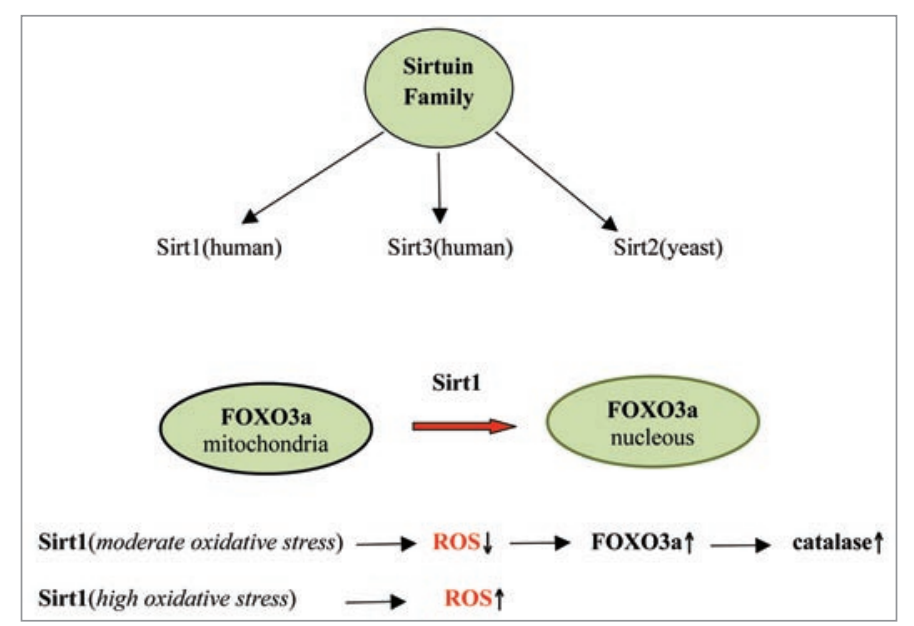

Figure 7. ROS-depended Sitruin pathways (Alcendor et al., ${ }^{39}$ Hasegawa et al. ${ }^{40}$ and Jacobs et al. ${ }^{41}$ ).

high levels of Sirt1 increased ROS formation in the heart (Fig. 7).

Hasegawa et al. $^{40}$ studied the role of Sirt1 in ROS-depended renal tubular cell apoptosis. Hydrogen peroxide induced FOXO3a nuclear accumulation and apoptosis while endogenous Sirt1 maintained cell survival by regulating catalase expression. Subauste and Burant showed that upregulation of Sirt1 stimulated the translocation of FOXO1 to the nucleus and increased FOXO1 protein levels in adipocytes exposed to FFA. ${ }^{38}$ Jacobs et al. ${ }^{41}$ found that Sirt3 forms a complex with FOXO3a in mitochondria. Expression of Sitr3 deacetylation mutant in cells led to an increase in intracellular superoxide production and oxidized glutathione. It was suggested that Sirt 3 and FOXO1 can comprise a mitochondrial signaling survival cascade.

KLOTHO gene. The Klotho gene was identified by Kuro-o et al. ${ }^{42}$ in 1997 . It has been shown at the beginning that Klotho controls the sensitivity of organism to insulin but later on this gene attracted particular attention for its involvment in aging processes. Klotho protein exists in two forms: the transmembrane form expressed primarily in renal tubular cells and the secreted form circulating in the blood. ${ }^{43}$

Yamamoto et al. ${ }^{44}$ studied signaling pathways through which Klotho increased resistance to oxidative stress. Cell surfacebound Klotho inhibited FOXO3a phosphorylation and promoted its nuclear translocation. The nuclear FOXO3a then 


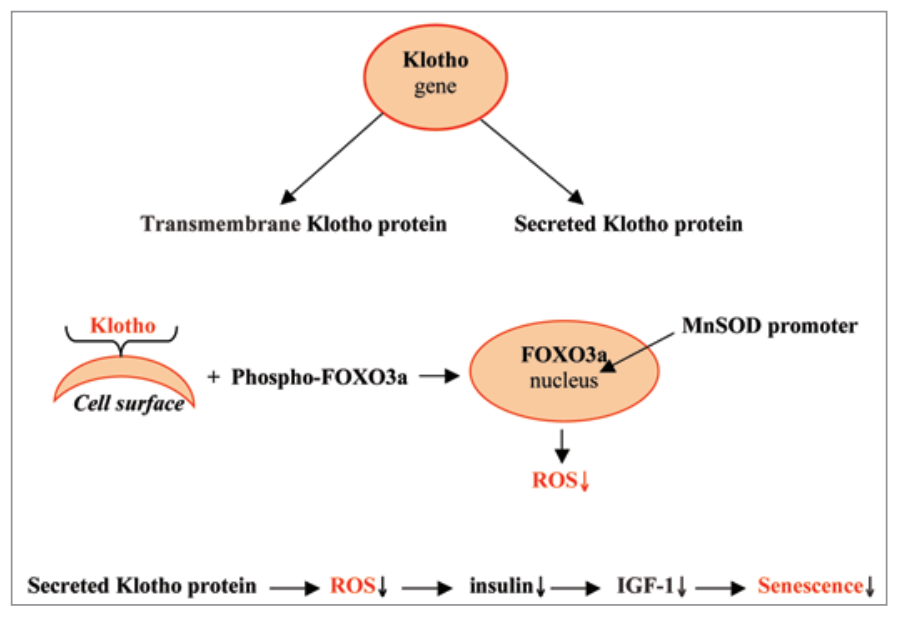

Figure 8. Mechanisms of Klotho antiaging effects (Kuro- $0,{ }^{43}$ and Yamamoto et al. ${ }^{44}$ ).

became bound to the MnSOD promoter and upregulated its expression, resulting suppression ROS formation. Thus Klotho protein can suppress aging trough two distinct mechanisms: the inhibition of insulinlike signaling and an increase in resistance to oxidative stress (Fig. 8).

Kurosu et al. ${ }^{45}$ demonstrated that the defect in Klotho gene expression in mice accelerated cellular senescence, on the other hand the overexpression of Klotho in mice extended life span. Administration of angiotensin II to rats decreased renal expression of klotho and caused abnormal iron deposition in renal cells. Saito et al. ${ }^{46}$ investigated the effects of iron overload and iron chelation on renal expression of klotho in untreated rats and rats treated with angiotensin II. Their findings suggested that iron overload and increased ROS overproduction were involved in the mechanism of angiotensin II-mediated modulation of klotho expression.

Ohta et al. ${ }^{47}$ investigated the effect of Klotho gene delivery on blood pressure and oxidative stress in vivo. They found that Klotho gene upregulated MnSOD expression and total SOD activity in the aorta of mice, enhanced nitric oxide production, and downregulated lipid peroxide concentration in serum of mice. It was concluded that klotho gene infusion into the tail vein of mice and rats suppressed ROS formation in animals.

Klotho overexpression in mice decreased hydrogen peroxide-induced apoptosis in endothelial cells and interfered with hydrogen peroxide-induced premature cellular senescence. ${ }^{48}$
Rakugi et al. ${ }^{49}$ studied the effects of membrane-bound Klotho on MnSOD expression and nitric oxide production in human umbilical vein endothelial cells (HUVEC). They found that the treatment of HUVEC with special klotho expression vectors enhanced MnSOD expression by approximately two-fold, partially through the activation of cAMP signaling pathway and increased nitric oxide production. In addition klotho inhibited angiotensin II-induced ROS production in HUVEC.

It has been shown that the Klotho gene is mainly expressed in the kidney tubules. Therefore the reduction of Klotho gene expression may contribute to the development of kidney failure while its overexpression may lead to the amelioration of renal injury. Haruna et al..$^{50}$ showed that the survival rate of old Klotho transgenic mice was approximately 30-70\% higher than that in wild-type mice. This improvement was associated with improvement in renal functions, cytochrome $c$ oxidase activity, and the reduction of superoxide generation. It was proposed that Klotho may serve as a circulating hormone capable of suppressing mitochondrial oxidative stress.

\section{Discussion}

Under normal physiological conditions the human organism tightly controls all the sources of ROS production and the damaging leak of ROS leading to various pathologies and aging should be minimal. However various external factors (environmental contamination, irradiation, etc.,), pathological disorders or toxic components of diet can stimulate cell senescence..$^{51}$ As it follows from the present consideration, ROS signaling is responsible for the initiation of gene- and enzyme-mediated cascades of senescence and aging. It is of utmost importance that reactive oxygen species are not only the initiators of these cascades but are also formed during these processes; owing to this, ROS signaling results in ROS overproduction and the acceleration of aging.

Contemporary studies demonstrate an astonishing phenomenon: a cooperation of ROS and aging-regulating genes in senescence and aging development. For a long time both ROS and genes were considered to be the important but separate stimuli of aging regulation, but it is now clear that they are interconnected. Numerous studies demonstrate the signaling functions of superoxide and hydrogen peroxide in various enzymatic heterolytic processes. ${ }^{2}$ Unfortunately at present, the mechanisms of ROS signaling in these reactions remains obscure. A rare example of the investigation of ROS signaling is the study of activation of protein kinase $\mathrm{C}$ by superoxide and hydrogen peroxide through the oxidation of thiol restudies and the release of zinc from the cysteine-rich region of the enzyme. ${ }^{52}$ Superoxide probably plays a critical role in PKC activation due to its ability to substitute zinc ion in the enzyme domain. However, in many other cases no suggestions have been made about the mechanism of ROS signaling in the enzymatic non-free radical processes.

It has been shown that ROS signaling in gene-regulated aging processes is mediated by enzymes. For example, lower survival of hepatocytes from aged rats was associated with reduced activations of ERK and Akt kinases, which protected against oxidant injury. ${ }^{53}$ Correspondingly, the inhibition of ERK and Akt activation in young cells markedly increased their sensitivity to hydrogen peroxide whereas caloric restriction (surviving factor) prevented loss in ERK and Akt activation and enhanced survival of old hepatocytes.

Jin et al. ${ }^{54}$ investigated ROS-dependent signal transduction pathways in the kidney of young and aged rats. Menadioneinduced PI3-kinase activity and Akt 
phosphorylation were significantly reduced in the old compared with the young. On the other hand, menadione-induced activities of JNK1 and AMPK were higher in the old rats. These findings suggest that the age-related downregulation of PI3kinase/Akt pathway and upregulation of JNK1 and AMPK enzymes are characteristics of ROS enhancement in aged rats.

Contradictory results concerning the participation of Akt kinase in ROSinitiated processes were recently received by Nogueira et al..$^{55}$ They demonstrated that although Akt kinase was usually regarded as a surviving factor, Akt deficiency caused resistance to replicative senescence, oxidative stress and ROSmediated apoptosis. At the same time Akt activation induced premature senescence and ROS-mediated apoptosis by increasing intracellular ROS through increased oxygen consumption and by inhibition of the expression of ROS scavengers downstream of FOXO. Akt kinase is a critical factor in the ROS-depended gene pathways of senescence/aging, therefore its double effects on ROS generation could be of utmost importance.

Another enzyme which is activated by ROS is AMP-activated protein kinase (AMPK). It has been proposed that mitochondrial ROS activate AMPK $\alpha 1$ in human umbilical vein endothelial cells (HUVECs) at a low oxygen concentration. Colombo and Moncada showed that AMPK $\alpha 1$ is responsible for the expression of antioxidant genes such as MnSOD, catalase, and others. ${ }^{56}$ Furthermore, peroxisome proliferator activated-coactivator-1 and FOXO3a are involved in this signaling pathway.

Thus we can see that ROS signaling is an important factor of enzyme and gene regulation of cell senescence and organismal aging. The above consideration demonstrates that there are many different pathways of stimulation and inhibition of senescence some of them are shown below:

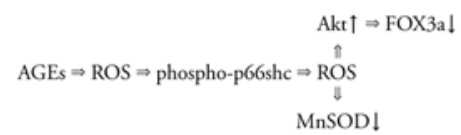

(Cai et al. ${ }^{16}$ )

HG $\downarrow \Rightarrow$ (phospho-Ser-36)p66shc $\downarrow \Rightarrow$ ROS $\downarrow \Rightarrow$ survival $\uparrow$ (Malhotra et al. ${ }^{17}$ )

$\mathrm{HG} \uparrow \Rightarrow$ IGF-1 $\Rightarrow$ phospho-Akt $\Rightarrow$ $\mathrm{ROS} \uparrow \Rightarrow$ FOXO3a $\downarrow\left(\right.$ Li et al. $\left.{ }^{32}\right)$
ERK1/Akt $\uparrow \Rightarrow$ survival $\uparrow$

$\operatorname{ROS}\left(\mathrm{H}_{2} \mathrm{O}_{2}\right) \Rightarrow$ ERK1/Akt $\downarrow \Rightarrow$ survival $\downarrow$ (Ikeyama, et al. ${ }^{53}$ )

ROS $\Rightarrow$ insulin/IGF $\Rightarrow$ PI $3 \uparrow \Rightarrow$ phospho-Akt $\uparrow \Rightarrow$ phospho-FOXO3a $\uparrow$ activation inactivation (Kim et al. ${ }^{29}$ )

Akt activation $\Rightarrow \operatorname{ROS} \uparrow \Rightarrow$ apoptosis $\uparrow$, senescence $\uparrow$ (Nogueira, et al..$^{58}$ )

ROS (moderated) $\Rightarrow$ EGFR/Ras/ MEK/ERK $\uparrow \Rightarrow$ survival $\uparrow$

ROS (severe) $\Rightarrow$ EGFR/Ras/MEK/ ERK $\downarrow \Rightarrow$ (phospho-Ser36)p66shc $\uparrow \Rightarrow$ senescence (Arany et al. ${ }^{23}$ )

$\alpha-1-\mathrm{AR} \Rightarrow$ PKC $\varepsilon$, PKC $\Rightarrow$ ROS $\uparrow$ $\Rightarrow$ phospho-p66shc $\Rightarrow$ phospho-Akt/ FOXO3a $\Rightarrow \mathrm{MnSOD} \downarrow \Rightarrow$ apoptosis $\uparrow$ (Guo et al. ${ }^{27}$ ).

Analysis of enzyme/gene pathways regulating aging development leads to following conclusions:

(1) ROS signaling is an obligatory stimulus of initiation and continuation of all studied processes.

(2) ROS-stimulated phosphorylation is an important factor of activation/inactivation of p66spc and $\mathrm{FOXO} 3 \mathrm{a}$ which causes cell senescence.

(3) Akt activation can both enhance and suppress senescence depending on the types of cells and other conditions.

(4) At least in one case ROS signaling resultes in both increasing and decreasing senescence depending on moderate or strong conditions of oxidative stress. ${ }^{23}$

How can we regulate ROS signaling in cellular senescence? Attempts to apply classic antioxidants such as ascorbic acid and $\alpha$-tocopherol for the treatment of healthy old subjects and experimental animals were not very successful. ${ }^{2}$ It can be expected if reactive oxygen species participating in aging processes are superoxide and hydrogen peroxide and not reactive free radicals ROO and $\mathrm{HO}$ because only the last two can be scavenged by such antioxidants. Superoxide is probably very important as the signaling species in aging development due to its high nucleophilic properties and ability to mediate heterolytic enzymatic reactions. This is supported by the fact that some signaling pathways leading to senescence retardation depends on upregulation of mitochondrial $\mathrm{MnSOD}$ - the scavenger of superoxide. Therefore, superoxide scavengers such as SOD mimetics could be potential effective aging suppressors. ${ }^{57-59}$ There are also other free radical scavengers which rapidly react with superoxide (flavonoids and ubiquinones), which can probably also be used as aging-retardant antioxidants. ${ }^{60,61}$

As is seen from the above, phosphorylation of the p66shc gene leads to its activation and the stimulation of senescence. On the other hand, phosphorylation of FOXO3a results in the inhibition of its anti-aging activity. It is of particular interest that the Sirtuin gene downregulated senescence at low ROS levels and stimulated it at high ROS levels. Klotho apparently always exhibits antioxidant effect. There could be major conclusions about pro- and anti-aging effects of p66hsc, FOXO3a, Sirtuin and Klotho genes.

In addition to gene regulation there is another factor capable of retarding aging development. It has been shown that the formation and bioavailability of nitric oxide was decreased in old human and animals. ${ }^{62-65}$ On the other hand, it has been shown that the Klotho gene enhanced NO formation in human and animal cells. ${ }^{47,49}$ Therefore, we can hypothesize that both anti-aging genes and nitric oxide are critical factors of aging development.

\section{References}

1. Afanas'ev I. Superoxide and nitric oxide in senescence and aging. Front Biosci 2009; 14:3899-912.

2. Afanas'ev IB. Signaling mechanisms of oxygen and nitrogen free radicals. 2009; Boca Raton, Fl., Taylor \& Francis CRC.

3. Afanas'ev IB. On mechanism of superoxide signaling under physiological and pathophysiological conditions. Med Hypotheses 2005; 64:127-9.

4. Baas AS, Berk BC. Differential activation of mitogenactivated protein kinases by $\mathrm{H}_{2} \mathrm{O}_{2}$ and $\mathrm{O}_{2}-$ in vascular smooth muscle cells. Circ Res 1995; 77:29-36.

5. Devadas S, Zaritskaya L, Rhee SG, Oberlay L, Williams MS. Discrete generation of superoxide and hydrogen peroxide by $\mathrm{T}$ cell receptor stimulation: Selective regulation of mitogen-activated protein kinase activation and Fas ligand expression. J Exp Med 2002; 195:59-70.

6. Moreno-Manzano V, Ishikawa Y, Lucio-Cazana J, Kitamura M. Selective involvement of superoxide anion, but not downstream compounds hydrogen peroxide and peroxynitrite, in tumor necrosis factoralpha-induced apoptosis of rat mesangial cells. J Biol Chem 2000; 275:12684-91

7. Cui XL, Douglas JG. Arachidonic acid activates c-jun $\mathrm{N}$-terminal kinase through NADPH oxidase in rabbit proximal tubular epithelial cells. Proc Natl Acad Sci USA 1997; 94:3771-6.

8. Lyng FM, Maguire P, McClean B, Seymour C, Mothersill $\mathrm{C}$. The involvement of calcium and MAP kinase signaling pathways in the production of radiation-induced bystander effects. Radiation Res 2006; 165:400-9. 
9. Migliaccio E, Giorgio M, Mele S, Pelicci G, Reboldi P, Pandolfi PP, et al. The p66shc adaptor protein controls oxidative stress response and life span in mammals. Nature 1999; 402:309-13.

10. Pinton P, Rimessi A, Marchi S, Orsini F, Migliaccio E, Giorgio $M$, et al. Protein kinase $C \beta$ and prolyl isomerase 1 regulate mitochondrial effects of the lifespan determinant p66Shc. Science 2007; 315:65963.

11. Gertz M, Fischer F, Wolters D, Steegborn C. Activation of the lifespan regulator p66Shc through reversible disulfide bond formation. Proc Natl Acad Sci USA 2008; 105:5705-9.

12. Orsini F, Migliaccio E, Moroni M, Contursi C, Raker VA, Piccini D, et al. The life span determinant p66Shc localizes to mitochondria where it associates with mitochondrial heat shock protein 70 and regulates trans-membrane potential. J Biol Chem 2004; 279:25689-95.

13. Trinei M, Giorgio M, Cicalese A, Barozzi S, Ventura A, Migliaccio E, et al. A p53-p66Shc signalling pathway controls intracellular redox status, levels of oxidation-damaged DNA and oxidative stressinduced apoptosis. Oncogene 2002; 21:3872-8.

14. Giorgio M, Migliaccio E, Orsini F, Paolucci D, Moroni M, Contursi C, et al. Electron transfer between cytochrome $c$ and p66Shc generates reactive oxygen species that trigger mitochondrial apoptosis. Cell 2005; 122:221-33.

15. Nemoto $S$, Combs CA, French $S$, Ahn B-H, Fergusson MM, Balaban RS, Finkel T. The mammalian longevity-associated gene product p66sho regulates mitochondrial metabolism. J Biol Chem 2006; 281:10555-60.

16. Cai W, He JC, Zhu L, Chen X, Striker GE, Vlassara H. AGE-receptor- 1 counteracts cellular oxidant stress induced by AGEs via negative regulation of p66shcdependent FKHRL1 phosphorylation. Am J Physiol Cell Physiol 2008; 294:145-52.

17. Malhotra A, Vashistha H, Yadav VS, Dube MG, Kalra SP, Abdellatif M, Meggs LG. Inhibition of p66ShcA redox activity in cardiac muscle cells attenuates hyperglycemia-induced oxidative stress and apoptosis. Am J Physiol Heart Circ Physiol 2009; 296:380-8.

18. Lebiedzinska M, Duszynski J, Rizzuto R, Pinton P, Wieckowski MR. Age-related changes in levels of p66Shc and serine 36-phosphorylated p66Shc in organs and mouse tissues. Arch Biochem Biophys 2009; 486:73-80

19. Khanday FA, Yamamori T, Mattagajasingh I, Zhang Z, Bugayenko, Naqvi A, et al. Rac1 leads to phosphorylation-dependent increase in stability of the p66shc adaptor protein: Role in Racl-induced oxidative stress. Mol Biol Cell 2006; 17:122-9.

20. Husain M, Meggs LG, Vashistha H, Simoes S, Griffiths KO, Kumar D, et al. Inhibition of p66ShcA longevity gene rescues podocytes from HIV-1induced oxidative stress and apoptosis. J Biol Chem 2009; 284:16648-58.

21. Berniakovich I, Trinei M, Stendardo M, Migliaccio E, Minucci S, Bernardi P, et al. p66Shc-generated oxidative signal promotes fat accumulation. J Biol Chem 2008; 283:34283-93.

22. Tomilov AA, Bicocca V, Schoenfeld RA, Giorgio M, Migliaccio E, Ramsey JJ, et al. Decreased superoxide production in macrophages of long-lived p66Shcknockout mice. J Biol Chem 2010; 285:1153-65.

23. Arany I, Faisal A, Nagamine Y, Safirstein RL. p66shc inhibits pro-survival epidermal growth factor receptor/ERK signaling during severe oxidative stress in mouse renal proximal tubule cells. J Biol Chem 2008; 283:6110-7.

24. Willcox BJ, Donlon TA, He Q, Chen R, Grove JS, Yano K, et al. FOXO3A genotype is strongly associated with human longevity. Proc Natl Acad Sci USA 2008; 105:13987-92.
25. Nemoto S, Finkel T. Redox regulation of forkhead proteins through a $p 66 s h c$-dependent signaling pathway. Science 2002; 295:2450-2.

26. Miyauchi H, Minamino T, Tateno K, Kunieda T, Toko H, Komuro I. Akt negatively regulates the in vitro lifespan of human endothelial cells via a p53/ p21-dependent pathway. EMBO J 2004; 23:212-20.

27. Guo J, Gertsberg Z, Ozden N, Steinberg SF. p66shc links \{alpha\}1-adrenergic receptors to a reactive oxygen species-dependent AKT-FOXO3A phosphorylation pathway in cardiomyocytes. Circ Res 2009; 104:660-9.

28. Purdom S, Chen QM. Linking oxidative stress and genetics of aging with p66Shc signaling and forkhead transcription factors. Biogerontology 2003; 4:181 91.

29. Kim HK, Kim YK, Song IH, Baek SH, Lee SR, Kim JH, Kim JR. Downregulation of a forkhead transcription factor, FOXO3a, accelerates cellula senescence in human dermal fibroblasts. J Gerontol A Biol Sci Med Sci 2005; 60:4-9.

30. Alikhani M, Maclellan CM, Raptis M, Vora S, Trackman PC, Graves DT. Advanced glycation end products induce apoptosis in fibroblasts through activation of ROS, MAP kinases, and the FOXO1 transcription factor. Am J Physiol Cell Physiol 2007; 292:850-6.

31. Kim DH, Kim JY, Yu BP, Chung HY. The activation of NFkappaB through Akt-induced FOXO1 phosphorylation during aging and its modulation by calorie restriction. Biogerontology 2008; 9:33-47.

32. Li M, Chiu JF, Mossman BT, Fukagawa NK. Downregulation of manganese-superoxide dismutase through phosphorylation of $\mathrm{FOXO} 3 \mathrm{a}$ by $\mathrm{Akt}$ in explanted vascular smooth muscle cells from old rats. J Biol Chem 2006; 281:40429-39.

33. Li M, Chiu JF, Gagne J, Fukagawa NK. Age-related differences in insulin-like growth factor-1 receptor signaling regulates $\mathrm{Akt} / \mathrm{FOXO} 3 \mathrm{a}$ and ERK/Fos pathways in vascular smooth muscle cells. J Cell Physio 2008; 217:377-87.

34. Collins AR, Lyon CL, Xia X, Liu JZ, Tangirala RK, Yin F, et al. Age-accelerated atherosclerosis correlates with failure to upregulate antioxidant genes. Circ Res 2009; 104:42-54

35. Tan WQ, Wang K, Lv DY, Li PF. Foxo3a inhibits cardiomyocyte hypertrophy through transactivating catalase. J Biol Chem 2008; 283:29730-9.

36. Nakamura T, Sakamoto K. Forkhead transcription factor FOXO subfamily is essential for reactive oxygen species-induced apoptosis. Mol Cell Endocrinol $2008 ; 281: 47-55$

37. Chintapalli J, Yang S, Opawumi D, Goyal SR, Shamsuddin N, Malhotra A, et al. Inhibition of wild type p66ShcA in mesangial cells prevents glycooxidant-dependent FOXO3a regulation and promotes the survival phenotype. Am J Physiol Renal Physiol 2007; 292:523-30.

38. Subauste AR, Burant CF. Role of FoxO1 in FFA induced oxidative stress in adipocytes. Am J Physio Endocrinol Metab 2007; 293:159-64.

39. Alcendor RR, Gao S, Zhai P, Zablocki D, Holle E, Yu $\mathrm{X}$, et al. Sirt1 regulates aging and resistance to oxidative stress in the heart. Circ Res 2007; 100:1512-21.

40. Hasegawa K, Wakino S, Yoshioka K, Tatematsu S, Hara Y, Minakuchi H, et al. Sirtl protects against oxidative stress-induced renal tubular cell apoptosis by the bidirectional regulation of catalase expression. Biochem Biophys Res Commun 2008; 372:51-6.

41. Jacobs KM, Pennington JD, Bisht KS, Aykin-Burn N, Kim HS, Mishra M, et al. SIRT3 interacts with the daf-16 homolog FOXO3a in the mitochondria, as well as increases FOXO3a dependent gene expression. Int J Biol Sci 2008; 4:291-9.

42. Kuro-o M, Matsumura Y, Aizawa H, Kawaguchi $\mathrm{H}$, Suga T, Utsugi T, et al. Mutation of the mouse klotho gene leads to a syndrome resembling ageing. Nature 1997; 390:45-51.
43. Kuro-o M. Klotho and aging. Biochim Biophys Acta 2009; 1790:1049-58

44. Yamamoto M, Clark JD, Pastor JV, Gurnani P, Nandi A, Kurosu H, et al. Regulation of oxidative stress by the anti-aging hormone Klotho. J Biol Chem 2005; 280:38029-34

45. Kurosu H, Yamamoto M, Clark JD, Pastor JV, Nandi A, Gurnani P, et al. Suppression of aging in mice by the hormone Klotho. Science 2005; 309:1829-33.

46. Saito K, Ishizaka N, Mitani H, Ohno M, Nagai R. Iron chelation and a free radical scavenger suppress angiotensin II-induced downregulation of klotho, an anti-aging gene, in rat. FEBS Lett 2003; 551:58-62.

47. Ohta J, Rakugi H, Ishikawa K, Yang J, Ikushima M, Chihara Y, et al. Klotho gene delivery suppresses oxidative stress in vivo. Geriat Gerontol Int 2007 7:293-9.

48. Ikushima M, Rakugi H, Ishikawa K, Maekawa Y, Yamamoto K, Ohta J, et al. Anti-apoptotic and antisenescence effects of Klotho on vascular endothelial cells. Biochem Biophys Res Commun 2006; 339:827-32.

49. Rakugi H, Matsukawa N, Ishikawa K, Yang J, Imai $M$, Ikushima $M$, et al. Anti-oxidative effect of Klotho on endothelial cells through cAMP activation. Endocrine 2007; 31:82-7.

50. Haruna Y, Kashihara N, Satoh M, Tomita N, Namikoshi T, Sasaki T, et al. Amelioration of progressive renal injury by genetic manipulation of Klotho gene. Proc Natl Acad Sci USA 2007; 104:2331-6.

51. Afanas'ev IB. Free radical mechanisms of aging processes under physiological conditions Biogerontology 2005; 6:283-90

52. Knapp LT, Klann E. Superoxide-induced stimulation of protein kinase $\mathrm{C}$ via thiol modification and modulation of zinc content. J Biol Chem 2000; 275:24136 45 .

53. Ikeyama S, Kokkonen G, Shack S, Wang X-T, Holbrook NJ. Loss in oxidative stress tolerance with aging linked to reduced extracellular signal-regulated kinase and Akt kinase activities. FASEB J 2001; 10.1096/fj.01-0409fje.

54. Jin Q, Jhun BS, Lee SH, Lee J, Pi Y, Cho YH, et al. Differential regulation of phosphatidyl 3-kinase/Akt, mitogen-activated protein kinase, and AMP-activated protein kinase pathways during menadione-induced oxidative stress in kidney of young and old rats. Biochem Biophys Res Commun 2004; 315:555-61.

55. Nogueira V, Park Y, Chen CC, Xu PZ, Chen ML, Tonic I, et al. Akt determines replicative senescence and oxidative or oncogenic premature senescence and sensitizes cells to oxidative apoptosis. Cancer Cell 2008; 14:458-70

56. Colombo SL, Moncada S. AMPKa1 regulates the antioxidant status of vascular endothelial cells. Biochem J 2009; 421:163-9.

57. Quick KL, Ali SS, Arch R, Xiong C, Wozniak D, Dugan LL. A carboxyfullerene SOD mimetic improves cognition and extends the lifespan of mice. Neurobiol Aging 2008; 29:117-28.

58. Clausen A, Doctrow S, Baudry M. Prevention of $\operatorname{cog}$ nitive deficits and brain oxidative stress with superoxide dismutase/catalase mimetics in aged mice. Neurobiol Aging 2008; 18.

59. Zhang HY, Doctrow SR, Xu L, Oberley LW, Beecher B, Morrison J, et al. Redox modulation of the liver with chronic antioxidant enzyme mimetic treatmen prevents age-related oxidative damage associated with environmental stress. FASEB J 2004; 18:1547-9.

60. Ishii N, Senoo-Matsuda N, Miyake M, Yasuda K, Ishii T, Hartman PS, Furukawa S. Coenzyme Q10 can prolong $C$. elegans lifespan by lowering oxidative stress. Mech Aging Dev 2004; 125:41-6. 
61. Ochoa JJ, Quiles JL, Lopes-Fria M, Huertas JR, Mataix J. Effect of lifelong coenzyme Q10 supplementation on age-related oxidative stress and mitochondrial function in liver and skeletal muscle of rats fed on a polyunsaturated fatty acid (PUFA)-rich diet. J Gerontol Ser A: Biol Sci Med Sci 2007; 62:1211-8.

62. Sun D, Huang A, Yan EH, Wu Z, Yan C, Kaminski PM, et al. Reduced release of nitric oxide to shear stress in mesenteric arteries of aged rats. Am J Physiol Heart Circ Physiol 2004; 286:2249-56.

63. Massimo C, Scarpelli P, Lorenzo ND, Caimi G, Orio FD, Ciancarelli MG. Impaired plasma nitric oxide availability and extracellular superoxide dismutase activity in healthy humans with advancing age. Life Sci 2006; 78:1163-7.

64. Chou T-C, Yen M-H, Li C-Y, Ding Y-A. Alterations of nitric oxide synthase expression with aging and hypertension in rats. Hypertension 1998; 31:643-8.

65. Payne JA, Reckelhoff JF, Khalil RA. Role of oxidative stress in age-related reduction of NO-cGMPmediated vascular relaxation in SHR. Am J Physiol Regul Integr Comp Physiol 2003; 285:542-51. 


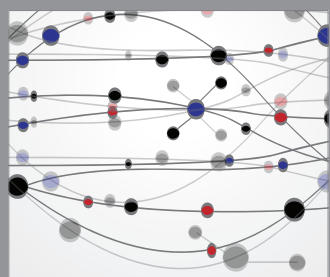

The Scientific World Journal
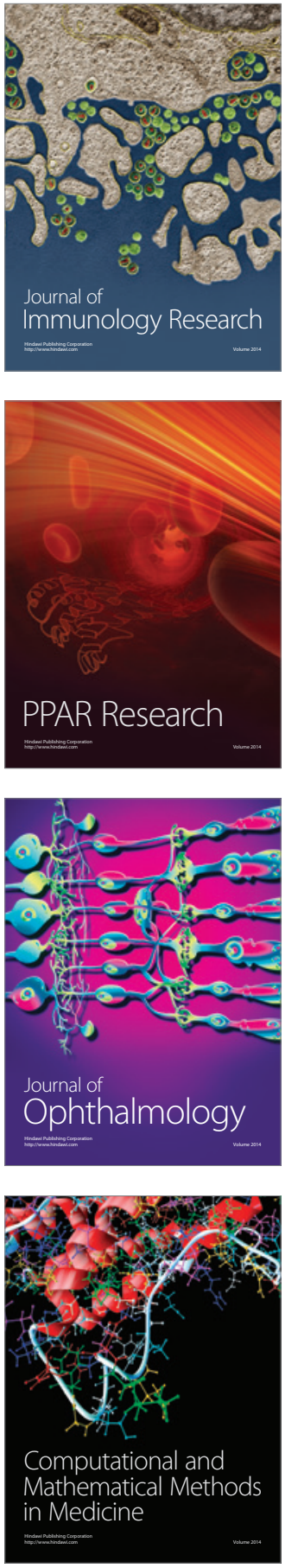

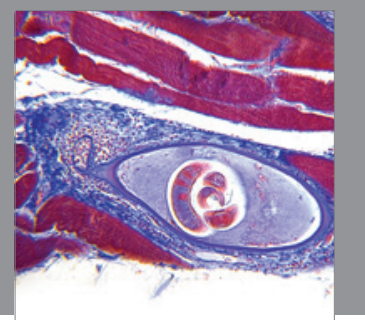

Gastroenterology

Research and Practice
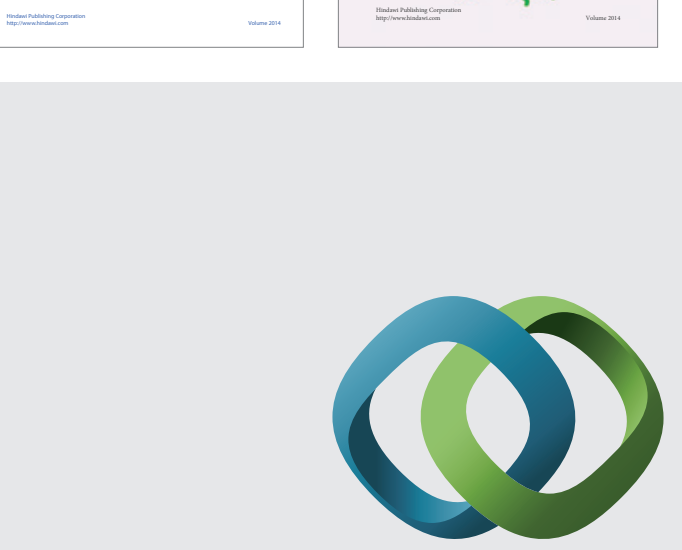

\section{Hindawi}

Submit your manuscripts at

http://www.hindawi.com
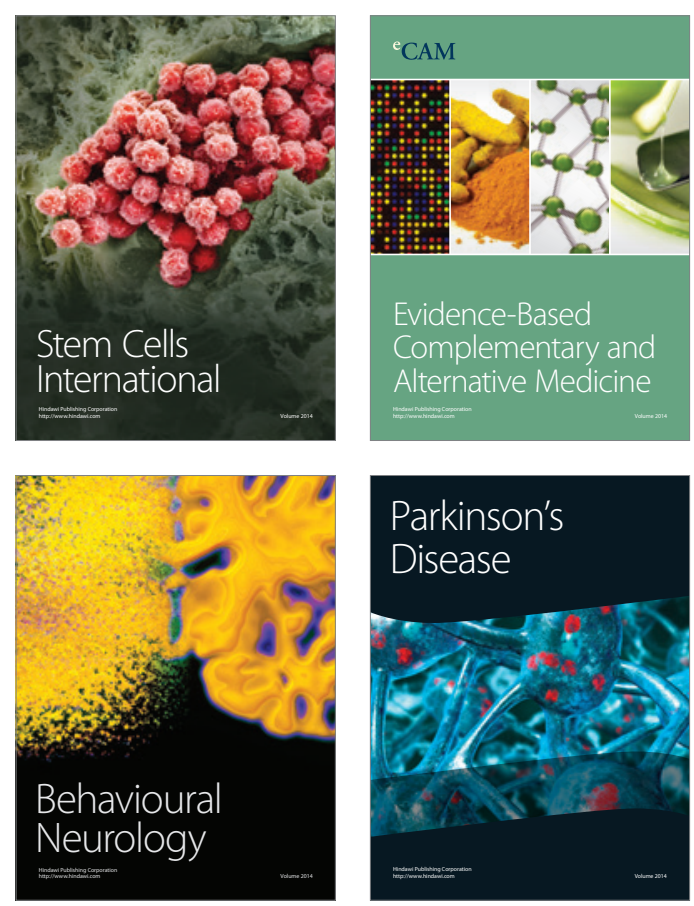

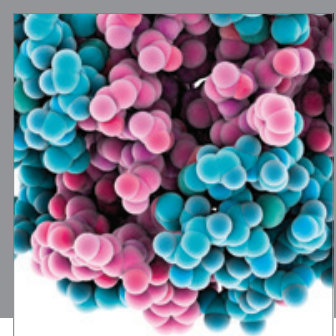

Journal of
Diabetes Research

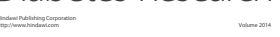

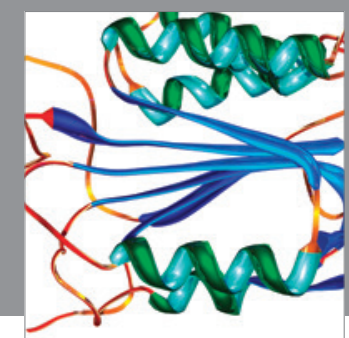

Disease Markers
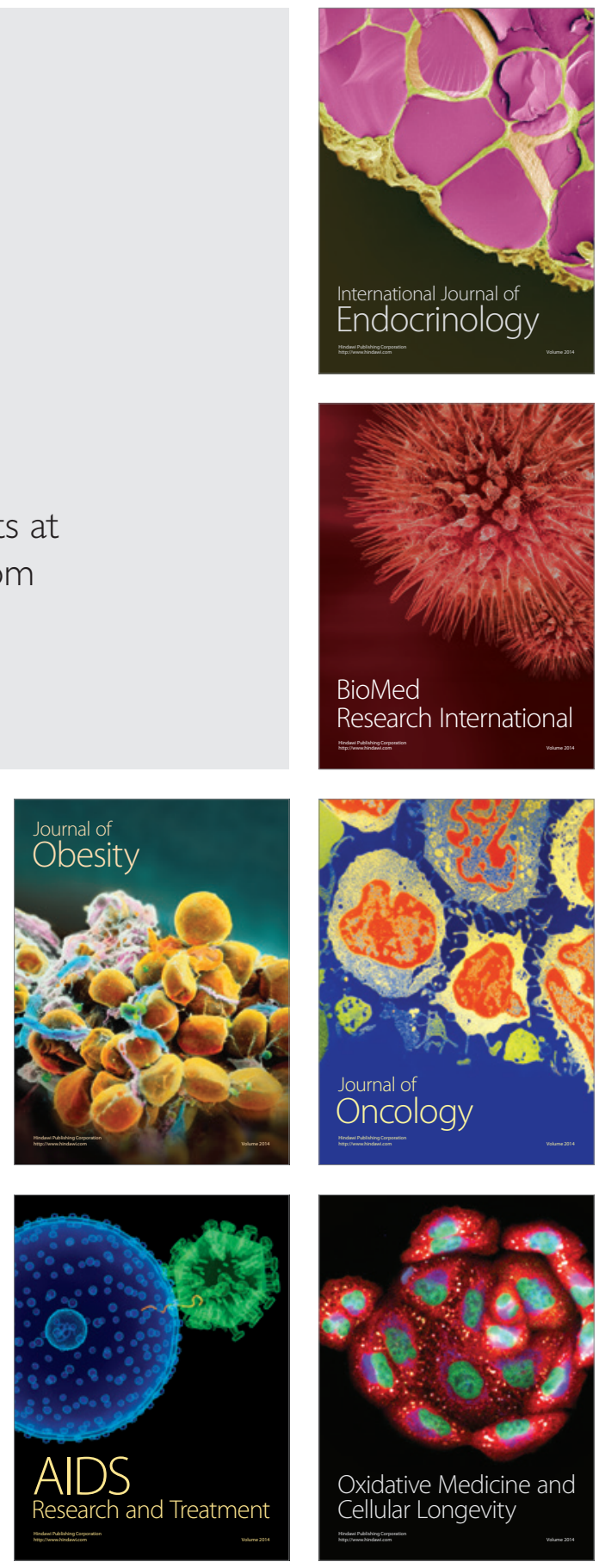